\title{
EVALUACIÓN DE MEDIDAS CORPORALES PARA LA SELECCIÓN DE LLAMAS MADRES Y CRÍAS
}

\author{
EVALUATION OF BODY MEASUREMENTS FOR THE SELECTION OF LAMAS AND THEIR BABIES
}

Víctor Leyva V. ${ }^{1}$ y Néstor Falcón P. ${ }^{2}$

\section{Resumen}

En las llamas y crías del núcleo reproductor del Centro Experimental IVITA-UNMSM se evaluó el peso corporal (PC), perímetro torácico (PT), las medidas del muslo (MU), grupa (GR) y ubre, y la producción de leche para determinar, a través de pruebas de correlación, los indicadores fenotípicos apropiados en la selección de llamas para producción de carne. La evaluación incluyó el efecto de la edad y paridad de la madre de años anteriores a través del análisis de varianza. En las medidas del muslo se consideró la circunferencia de su perímetro superior (CSMU) e inferior (CIMU) y su longitud (LMU); y en la grupa, la distancia entre sus tuberosidades isquiática medial, lateral, sacra y coxal. En la ubre se consideró su longitud y ancho y el perímetro del área entre pezones (PAP), registradas previo al ordeño. El volumen de leche fue medido cada semana hasta la semana 7 de lactación y simultáneo a estas medidas se obtuvo el peso corporal de la madre y crías. La paridad y edad afectó el PC, PT y todas las medidas de la ubre; y en las medidas del muslo solo hubo efecto de la edad. En las madres, hubo correlación significativa (alta y media) de su PC con PT, ancho de la ubre y PAP con todas las medidas del muslo y de la grupa. En la producción de leche, no hubo efecto de la paridad y la edad y en su relación con las diferentes variables, destacaron la correlación media alta con el ancho de la ubre $(\mathrm{p}<0.001)$ y media baja con el PAP $(\mathrm{p}<0.1)$. En la cría, hubo correlación alta y significativa entre PC, edad y PT y de LMU del muslo con CSMU y CIMU; con la edad su relación fue inversamente proporcional. En la relación madre-cría, las variables paridad, edad, PC, ancho de ubre, PAP y producción de leche tuvieron relaciones positivas con PC de las crías; similar patrón se tuvo con PT, a excepción de la paridad y edad, incluso con PT y CSMU e CIMU de la madre. La CSMU y CIMU y LMU de la cría solo tuvo correlación media con las medidas de la ubre. En conclusión, las variables asociados a la producción de leche (largo, ancho y PAP de la ubre) son las más importantes para determinar crías de mejor peso y, por lo tanto, pueden ser consideradas como criterios de selección de las madres con la finalidad de obtener crías de alto valor fenotípico. Aunado a ellos, las variables PC de la madre, y la CSMU y CIMU del muslo deben de ser incluidas para la selección de animales élite.

Palabras clave: llama, peso corporal, medidas corporales, ubre, valor fenotípico

${ }^{1}$ Laboratorio de Reproducción Animal, FMV-UNMSM

${ }^{2}$ Laboratorio de Medicina Veterinaria Preventiva, FMV-UNMSM 


\section{Abstract}

Lamas and their babies from the IVITA Research Center of San Marcos University were used to evaluate the body weight (BW), thoracic perimeter (TP), measures of thigh (TH), rump (RU) and udder, and milk yield, through correlation test to determine the suitable phenotype indicators for the selection of animals for meat production. The evaluation included the effect of age and parity of the dams from previous years using the ANOVA analysis. In the thigh was measured the length (LTH) and the circumference of its upper (CSTH) and lower (CITH) perimeter and, on the rump, the distance between its medial, lateral, sacral and coxal tuberosities. In the udder was measured the length, width and perimeter of the area between teats (PAT). The milk yield was weekly recorded until week seven of lactation and simultaneous to these measures was obtained the body weight of both mothers and babies. There were age and parity effects on TP and udder measurements; however, only the former effect was evident on thigh measurement. There was a significant relationship (high and mediun) of BW with TP, udder width and PAT with all thigh and rump measurements. Milk yield was not affected by age and parity, however had a clear relationship with udder width $(\mathrm{p}<0.01)$ and PAT $(\mathrm{p}<0.1)$. In babies was found a high and significant relationship of BW with age and TP, LTH with CSTH and CITH while had an inverse relationship with age. In the mother-baby interaction, parity, age, BW, udder width, PAT and mother milk yield had positive relationships with baby BW; similar pattern had with TP, with the exception of parity and age, even with mother PT, CSTH and CITH. The CSTH and CITH and length of baby thigh had only a mediun relationship with udder measurements. In conclusion, the variables associated with milk yield (length and width of the udder and the PAT) were the most important to determine BW of baby lamas, which allow the selection of lama mothers to produce babies of high phenotypic value. The variables BW of the mother and the CSTH and CITH must be considered to select superior animals.

Key words: lama, body weight, body measurements, udder, phenotypic value

\section{INTRODUCCIÓN}

La crianza de la llama en el Perú se realiza mayormente en conjunto con otras especies, donde la población de llamas es del 5$10 \%$. El objetivo productivo de su crianza no está claramente definido, pero se le usa extensivamente como animal de carga y sólo se les beneficia a una edad avanzada (Leyva, 1989; Proyecto Camélidos Sudamericanos, 1990). Esta práctica, asociada a su alimentación en pradera nativa con limitada calidad de pasto, sobre todo en la época seca (San Martín, 1996), resulta en una carcasa de calidad magra y textura inapropiada, cualidades que limitan su comercialización.
La llama tiene un 58 a $60 \%$ de rendimiento de carcasa (Bravo et al., 1981), en tanto que la alpaca tiene 52\% (Calderón y Fernández Baca, 1972) y el ovino criollo tiene 40\% (Fernández Baca, 1961). Además, presenta una mayor eficiencia digestiva en la utilización de pastura de baja calidad y en la conversión del alimento consumido, con un menor requerimiento para mantenimiento, comparado con el de ovino (San Martín, 1996). Estas cualidades indican que esta especie posee capacidad para responder con eficiencia a programas de manejo nutricional para producción de carne.

La propuesta de orientar la llama para producción de carne requiere del estableci- 
miento de un núcleo de reproductores con el adecuado potencial genético; sin embargo, para poder seleccionar a los mejores animales se requiere conocer los caracteres fenotípicos relacionados con la expresión de esta capacidad genética a fin de utilizarlos como indicadores fenotípicos.

El peso corporal es un indicador ampliamente utilizado, pero no expresa necesariamente la condición cárnica. Otras características que han sido estudiadas incluyen el perímetro torácico (Condori et al., 2003), largo de la grupa (Estrada, 1983), distancia entre puntas de las caderas (Bustinza et al., 1993), ancho de ancas y la altura a la cruz (Condori et al., 2003). Así mismo, la condición corporal en llamas es una medida que refleja su estado nutricional (Johnson, 1994; Pugh, 1997).

La zona del muslo y de la grupa constituye el 35\% de la carne del ovino carnicero; sin embargo, se desconoce esta información en la llama. El presente estudio tuvo por objetivo determinar el tamaño de varias zonas corporales, y la comparación de estas variables entre la madre y la cría con el fin de utilizarlos como indicadores fenotípicos para la selección de esta especie.

\section{Materiales y Métodos}

El estudio se realizó en la estación experimental del Centro de Investigación IVITA, de la Facultad de Medicina Veterinaria, Universidad Nacional Mayor de San Marcos, ubicado en el distrito de Maranganí, provincia de Canchis, departamento de Cusco. La zona de crianza tiene una altitud aproximada de 4,338 msnm, una precipitación pluvial de 900 $\mathrm{mm}$ al año y una temperatura anual promedio de $6.5^{\circ} \mathrm{C}$.

Se utilizaron las llamas con cría del núcleo reproductor de la campaña de parición 2005. La información sobre la edad y número de parto se obtuvo de los registros del centro experimental. Los animales pastoreaban praderas nativas. Las especies de pastos nativos en zonas secas fueron Festuca dolichophyla, Muhlembergia fastigiate, Stipa brachy-phyla y Alchemilla pinnata, entre otras, y los pastos de zonas húmedas fueron Distichia muscoides, Scirpus rigidus y Plantago tubulosa.

Los animales estaban identificados con aretes metálicos que fueron colocados al nacimiento y con pintura en la cabeza para facilitar la detección por clase y grupos dentro del rebaño. Todos estuvieron sujetos a los programas de manejo y sanidad de la estación experimental.

El peso corporal se registró en ayunas. El perímetro torácico se midió tomando como puntos anatómicos de referencia la cruz y la parte anterior del torax (inmmediatamente detrás de la articulación del húmero con el omóplato). En las medidas del muslo se consideró la circunferencia del perímetro superior e inferior y la línea interna del muslo. Para la primera se tomó como punto de referencia la altura de la inserción del pliegue de la babilla y la línea inguinal y para la segunda la circunferencia inmediatamente encima de la articulación fémoro-tibio-rotuliana. En la grupa se tomó como referencia las tuberosidades isquiática medial (A), sacra, (B), coxal (C) e isquiática lateral (D) y se determinó las medidas de las distancias entre ellas (A-B, B-C, C-D y AD), de acuerdo a lo establecido por Zea $e t$ al. (2007). Todos los parámetros fueron registrados simultáneamente el mismo día entre las 06:00 y las 10:00 horas.

La producción de leche se evaluó semanalmente hasta la $7^{\text {ma }}$ semana de lactación. Para estimar la producción de leche, se colocó un protector de ubre (Leyva y Markas, 1991) por 4 horas (05:00-09:00) y luego se dejó amamantar a la cría para vaciar la ubre; luego se volvió a colocar el protector de ubre (09:00-13:00) para dejar que se llene. Allí se aplicó 8 UI de oxitocina (Leyva et al., 1983a,b) e inmediatamente se realizó el ordeño a mano hasta vaciar por completo la ubre. Consecuentemente, la pro- 
Cuadro 1. Edad (años), peso corporal $(\mathrm{kg})$, perímetro torácico $(\mathrm{cm})$, medidas del muslo y de la ubre $(\mathrm{cm})$ y producción de leche $(\mathrm{ml})$ de llamas (promedio \pm desviación estándar), según el número de partos

\begin{tabular}{|c|c|c|c|c|c|c|}
\hline & \multicolumn{5}{|c|}{ Número de parto } & \multirow{2}{*}{ Total } \\
\hline & 1 & 2 & 3 & 4 & 5 & \\
\hline Edad & $\begin{array}{c}3.7 \pm 1.8^{\mathrm{a}} \\
(19)\end{array}$ & $\begin{array}{c}4.7 \pm 0.8^{b} \\
(19)\end{array}$ & $\begin{array}{c}5.9 \pm 0.7^{\mathrm{c}} \\
(15)\end{array}$ & $\begin{array}{c}6.4 \pm 1.3^{c} \\
\quad(12)\end{array}$ & $\begin{array}{c}8.6 \pm 1.5^{\mathrm{d}} \\
\quad(12)\end{array}$ & $\begin{array}{l}5.6 \pm 2.1 \\
\quad(77)\end{array}$ \\
\hline $\begin{array}{l}\text { Peso } \\
\text { corporal }\end{array}$ & $\begin{array}{c}88.3 \pm 11.1^{\mathrm{a}} \\
(18)\end{array}$ & $\begin{array}{c}85.5 \pm 8.3^{\mathrm{a}} \\
(19)\end{array}$ & $\begin{array}{c}86.4 \pm 8.6^{\mathrm{a}} \\
(15)\end{array}$ & $\begin{array}{c}87.0 \pm 10.5^{\mathrm{a}} \\
(12)\end{array}$ & $\begin{array}{c}97.6 \pm 10^{b} \\
(12)\end{array}$ & $\begin{array}{c}81.5 \pm 10.3 \\
(76)\end{array}$ \\
\hline $\begin{array}{l}\text { Perímetro } \\
\text { torácico }\end{array}$ & $\begin{array}{c}110.9 \pm 4.6^{\mathrm{a}} \\
(18)\end{array}$ & $\begin{array}{c}109.9 \pm 4.6^{\mathrm{a}} \\
(19)\end{array}$ & $\begin{array}{c}109.5 \pm 4.3^{\mathrm{a}} \\
(15)\end{array}$ & $\begin{array}{c}112.5 \pm 5.8^{\mathrm{a}} \\
(12)\end{array}$ & $\begin{array}{c}116.8 \pm 4.4^{\mathrm{b}} \\
(12)\end{array}$ & $\begin{array}{c}111.6 \pm 5.2 \\
(76)\end{array}$ \\
\hline $\begin{array}{r}\text { Muslo } \\
\text { PS } \\
\text { PI } \\
\text { A }\end{array}$ & $\begin{array}{c}61.5 \pm 3.2^{\mathrm{a}} \\
40.8 \pm 2.6^{\mathrm{a}} \\
28.8 \pm 1.5^{\mathrm{a}} \\
(18)(16)^{1}\end{array}$ & $\begin{array}{c}60.0 \pm 3.5^{\mathrm{a}} \\
40.0 \pm 2.9^{\mathrm{a}} \\
29.0 \pm 4.5^{\mathrm{a}} \\
(19)(18)^{1}\end{array}$ & $\begin{array}{c}59.7 \pm 3.2^{\mathrm{a}} \\
39.7 \pm 3.0^{\mathrm{a}} \\
28.2 \pm 1.7^{\mathrm{a}} \\
(15)(14)^{1}\end{array}$ & $\begin{array}{c}61.4 \pm 2.1^{\mathrm{a}} \\
41.5 \pm 2.6^{\mathrm{a}} \\
29.1 \pm 1.0^{\mathrm{a}} \\
\quad(12)\end{array}$ & $\begin{array}{c}62.2 \pm 3.5^{\mathrm{a}} \\
41.9 \pm 3.1^{\mathrm{a}} \\
29.1 \pm 1.9^{\mathrm{a}} \\
(12)\end{array}$ & $\begin{array}{l}60.9 \pm 3.2 \\
40.7 \pm 2.9 \\
28.8 \pm 2.6 \\
(76)(72)^{1}\end{array}$ \\
\hline $\begin{array}{l}\text { Ubre } \\
\qquad \begin{array}{l}\text { L } \\
\text { A } \\
\text { PAP }\end{array}\end{array}$ & $\begin{array}{c}21.3 \pm 2.1^{\mathrm{a}} \\
19.3 \pm 1.6^{\mathrm{a}} \\
18.0 \pm 2.7^{\mathrm{a}} \\
\quad(19)\end{array}$ & $\begin{array}{c}21.2 \pm 1.7^{\mathrm{a}} \\
19.1 \pm 1.8^{\mathrm{a}} \\
18.6 \pm 1.9^{\mathrm{a}} \\
\quad(18)\end{array}$ & $\begin{array}{c}22.3 \pm 2.1^{\mathrm{a}} \\
19.6 \pm 2.7^{\mathrm{a}} \\
17.8 \pm 2.4^{\mathrm{a}} \\
(14)\end{array}$ & $\begin{array}{c}22.0 \pm 1.6^{\mathrm{a}} \\
19.7 \pm 2.6^{\mathrm{a}} \\
16.9 \pm 2.6^{\mathrm{a}} \\
(12)\end{array}$ & $\begin{array}{c}22.5 \pm 1.8^{\mathrm{a}} \\
20.1 \pm 2.6^{\mathrm{a}} \\
18.1 \pm 2.4^{\mathrm{a}} \\
\quad(12)\end{array}$ & $\begin{array}{c}21.8 \pm 0.9 \\
19.5 \pm 2.2 \\
18.0 \pm 2.4 \\
(75)\end{array}$ \\
\hline $\begin{array}{l}\text { Producción } \\
\text { de leche }\end{array}$ & $\begin{array}{c}234 \pm 67^{\mathrm{a}} \\
(6)\end{array}$ & $\begin{array}{c}233 \pm 42^{\mathrm{a}} \\
\quad(9)\end{array}$ & $\begin{array}{c}265 \pm 92^{\mathrm{a}} \\
\quad(10)\end{array}$ & $\begin{array}{c}249 \pm 62^{\mathrm{a}} \\
(8)\end{array}$ & $\begin{array}{l}- \\
-\end{array}$ & $\begin{array}{c}247 \pm 68 \\
\quad(33)\end{array}$ \\
\hline
\end{tabular}

ducción de leche registrada corresponde a la producción de 4 horas.

Las medidas de la ubre se registraron antes del ordeño. Se tomó como referencias la base de implantación de la ubre para medir la línea longitudinal que separa los cuartos derechos de los izquierdos y la transversal que separa los cuartos anteriores de los posteriores; además, se midió la distancia entre pezones de los cuartos anteriores (a), posteriores (p), laterales derecho (d) e izquierdo (i), y la suma de estas medidas (APDI) constituyó el perímetro del área de los pezones.
En el pesaje de los animales se utilizó una balanza metálica de reloj con una capacidad máxima de $250 \mathrm{~kg}$ y una precisión de $0.250 \mathrm{~kg}$. La balanza se colocó en un trípode metálico provisto de gancho y fajas para la sujeción y suspensión de las llamas. Para el registro de las medidas del perímetro torácico, muslo, grupa y ubre se utilizó la cinta métrica Goldfish de $150 \mathrm{~cm}$. Las llamas fueron ordeñadas a mano y la leche se midió en buretas graduadas de $250 \mathrm{ml}$.

Se utilizó el análisis de varianza (SAS, 1996) para evaluar la diferencia existente en- 
Cuadro 2. Peso corporal $(\mathrm{kg})$, perímetro torácico $(\mathrm{cm})$, medidas del muslo y de la ubre $(\mathrm{cm})$ y producción de leche $(\mathrm{ml})$ de llamas (promedio \pm desviación estándar), según la edad

\begin{tabular}{|c|c|c|c|c|c|}
\hline & \multicolumn{4}{|c|}{ Edad (años) } & \multirow{2}{*}{ Total } \\
\hline & $<3.5$ & 3.5 a 5 & $>5$ a 7 & $>7$ & \\
\hline Peso vivo & $\begin{array}{c}88.0 \pm 10.8^{\mathrm{a}} \\
(15)\end{array}$ & $\begin{array}{c}82.8 \pm 7.6^{\mathrm{a}} \\
(15)\end{array}$ & $\begin{array}{c}87.1 \pm 8.8^{\mathrm{a}} \\
(31)\end{array}$ & $\begin{array}{c}97.6 \pm 9.9^{b} \\
(15)\end{array}$ & $\begin{array}{c}88.5 \pm 10.3 \\
(76)\end{array}$ \\
\hline $\begin{array}{l}\text { Perímetro } \\
\text { torácico }\end{array}$ & $\begin{array}{c}110.7 \pm 4.7^{\mathrm{a}} \\
(15)\end{array}$ & $\begin{array}{c}108.5 \pm 4.1^{\mathrm{a}} \\
(15)\end{array}$ & $\begin{array}{c}110.8 \pm 4.5^{\mathrm{a}} \\
(31)\end{array}$ & $\begin{array}{c}117.1 \pm 4.4^{b} \\
(15)\end{array}$ & $\begin{array}{c}111.6 \pm 5.2 \\
(76)\end{array}$ \\
\hline \multicolumn{6}{|l|}{ Muslo } \\
\hline PS & $61.8 \pm 3.1^{\mathrm{a}}$ & $59.6 \pm 3.1^{\mathrm{ab}}$ & $60.2 \pm 3.0^{\mathrm{bc}}$ & $62.6 \pm 3.3^{c}$ & $60.9 \pm 3.2$ \\
\hline PI & $40.7 \pm 2.7^{\mathrm{a}}$ & $39.5 \pm 2.6^{\mathrm{ab}}$ & $40.5 \pm 2.9^{\mathrm{ab}}$ & $42.2 \pm 2.9^{\mathrm{b}}$ & $40.7 \pm 2.9$ \\
\hline $\mathrm{A}$ & $\begin{array}{l}28.9 \pm 1.4^{\mathrm{a}} \\
(15)(14)^{1}\end{array}$ & $\begin{array}{c}28.1 \pm 1.7^{\mathrm{a}} \\
(15)(14)^{1}\end{array}$ & $\begin{array}{c}29.1 \pm 3.5^{\mathrm{a}} \\
(31)(30)^{1}\end{array}$ & $\begin{array}{c}29.0 \pm 1.9^{\mathrm{a}} \\
(15)\end{array}$ & $\begin{array}{l}28.8 \pm 2.6 \\
(76)(73)^{1}\end{array}$ \\
\hline \multicolumn{6}{|l|}{ Ubre } \\
\hline $\mathrm{L}$ & $21.1 \pm 2.0^{\mathrm{a}}$ & $21.2 \pm 1.6^{\mathrm{a}}$ & $22.3 \pm 1.9^{\mathrm{a}}$ & $22.2 \pm 1.8^{\mathrm{a}}$ & $21.8 \pm 1.9$ \\
\hline A & $19.1 \pm 1.5^{\mathrm{a}}$ & $18.8 \pm 1.9^{\mathrm{a}}$ & $20.2 \pm 2.4^{\mathrm{a}}$ & $19.5 \pm 2.3^{\mathrm{a}}$ & $19.5 \pm 2.2$ \\
\hline PAP & $\begin{array}{c}17.8 \pm 2.1^{\mathrm{a}} \\
(15)\end{array}$ & $\begin{array}{c}18.3 \pm 1.7^{\mathrm{a}} \\
(16)\end{array}$ & $\begin{array}{c}17.8 \pm 2.9^{\mathrm{a}} \\
(29)\end{array}$ & $\begin{array}{c}17.9 \pm 2.4^{\mathrm{a}} \\
(15)\end{array}$ & $\begin{array}{l}18.0 \pm 2.4 \\
\quad(75)\end{array}$ \\
\hline $\begin{array}{l}\text { Producción } \\
\text { de leche }\end{array}$ & $\begin{array}{c}199 \pm 80^{\mathrm{a}} \\
(5)\end{array}$ & $\begin{array}{c}248 \pm 61^{\mathrm{a}} \\
(6)\end{array}$ & $\begin{array}{c}258 \pm 72^{\mathrm{a}} \\
(17)\end{array}$ & $\begin{array}{c}257 \pm 34^{\mathrm{a}} \\
(5)\end{array}$ & $\begin{array}{c}247 \pm 68 \\
\quad(33)\end{array}$ \\
\hline
\end{tabular}

tre las medidas zoométricas, peso vivo, perímetro torácico, medidas de la grupa, perímetro superior e inferior y longitud del muslo de la madre y su cría con relación a la edad y número de parto de la madre, y las diferencias en el largo, ancho de la ubre y perímetro del área de los pezones de la ubre de la madre con la tasa de crecimiento de la cría.

La prueba de correlación de Pearson (SAS, 1996) fue usada para determinar la fuerza y dirección en las que se encuentran las variables zoométricas entre las madres, entre las crías y las correlaciones correspondientes a madre-cría.

\section{Resultados}

El Cuadro 1 muestra la variabilidad de la edad, peso corporal, perímetro torácico, medidas del muslo y de la ubre y producción de leche de las llamas bajo estudio. Se observa que las primerizas iniciaron tardíamente su vida reproductiva ( $>3$ años de edad). El peso corporal fue similar entre las llamas de primer y cuarto parto, y solamente las madres de $5^{\text {to }}$ parto tuvieron un peso superior $(\mathrm{p}<0.05)$. El perímetro torácico tuvo un comportamiento similar. Sin embargo, no se encontró diferencia significativa entre 
Cuadro 3. Correlaciones significativas entre algunas variables productivas y medidas zoométricas de llamas madres

\begin{tabular}{lccc}
\hline Variables & $\begin{array}{c}\text { Madres } \\
(\mathrm{n})\end{array}$ & $\begin{array}{c}\text { Correlación } \\
(\mathrm{r})\end{array}$ & $\begin{array}{c}\text { Nivel de } \\
\text { significancia }\end{array}$ \\
\hline Peso corporal - Parto & 76 & 0.245 & 0.033 \\
Peso corporal - Edad & 78 & 0.412 & 0.000 \\
Peso corporal - Perímetro torácico & 77 & 0.813 & 0.000 \\
Peso corporal - Altura del muslo & 73 & 0.261 & 0.026 \\
Peso corporal - Perímetro superior del muslo & 77 & 0.706 & 0.000 \\
Peso corporal - Perímetro inferior del muslo & 77 & 0.638 & 0.000 \\
Peso corporal - Ancho de ubre & 77 & 0.347 & 0.002 \\
Peso corporal - Perímetro área pezones & 77 & 0.316 & 0.005 \\
Peso corporal - Medida AB & 77 & 0.216 & 0.059 \\
Peso corporal - Medida AD & 77 & 0.242 & 0.034 \\
Peso corporal - Medida CB & 77 & 0.367 & 0.001 \\
Peso corporal - Medida CD & 77 & 0.442 & 0.000 \\
Altura del muslo - Perímetro torácico & 73 & 0.369 & 0.001 \\
Altura del muslo - Perímetro superior del & 73 & 0.313 & 0.007 \\
$\quad$ muslo & 73 & 0.263 & 0.025 \\
Altura del muslo - Perímetro inferior del & 73 & 0.748 & 0.000 \\
muslo & & 0.507 & 0.000 \\
Altura del muslo - Medida AD & 73 & 0.433 & 0.000 \\
Altura del muslo - Medida CD & 73 & & \\
\hline AB: distanca ente la to & & & \\
\hline
\end{tabular}

$A B$ : distancia entre la tuberosidad isquiática medial y la sacra; $A D$ : entre la tuberosidad isquiática medial y la isquiática lateral; CB: entre la tuberosidad coxal y la sacra; CD: entre la tuberosidad coxal e isquiática lateral

las medidas del muslo y la ubre ni entre la producción de leche por efecto del número de partos.

En el Cuadro 2 se observa el efecto del grupo etario sobre el peso corporal, perímetro torácico, medidas del muslo y de la ubre y producción de leche de las llamas. El peso y el perímetro torácico de las madres no varió significativamente por efecto de la edad, con excepción de los animales $>7$ años que tuvieron un peso superior ( $\mathrm{p}<0.05$, Cuadro 2$)$. Tampoco se encontró diferencias significativas entre las medidas de la ubre ni de la producción de leche por efecto de la edad. Los perímetros superior e inferior del muslo se incrementaron con la edad del animal ( $<<0.05)$, mas no así la altura del muslo. La producción de leche de las madres tampoco se afectó por efecto de la edad de los animales. 
Cuadro 4. Estadística descriptiva de las variables de medición en las crías de llamas, según edad (meses)

\begin{tabular}{lccc}
\hline Variable & $\begin{array}{c}1 \text { mes } \\
(\mathrm{n}=22)\end{array}$ & $\begin{array}{c}2 \text { meses } \\
(\mathrm{n}=69)\end{array}$ & $\begin{array}{c}3 \text { meses } \\
(\mathrm{n}=80)\end{array}$ \\
\hline Peso & $16.8 \pm 2.32^{\mathrm{a}}$ & $21.4 \pm 3.26^{\mathrm{b}}$ & $27.2 \pm 4.29^{\mathrm{c}}$ \\
Perímetro torácico & $59.6 \pm 3.87^{\mathrm{a}}$ & $63.5 \pm 4.11^{\mathrm{b}}$ & $69.2 \pm 3.59^{\mathrm{c} 1}$ \\
Perímetro superior del muslo & $39.6 \pm 3.59^{\mathrm{a}}$ & $39.5 \pm 2.94^{\mathrm{a}}$ & $39.1 \pm 4.48^{\mathrm{a}}$ \\
Perímetro inferior del muslo & $29.2 \pm 2.66^{\mathrm{a}}$ & $29.1 \pm 1.95^{\mathrm{a}}$ & $29.0 \pm 3.00^{\mathrm{a}}$ \\
Altura del muslo & $20.8 \pm 1.70^{\mathrm{a}}$ & $19.8 \pm 1.05^{\mathrm{b}}$ & $19.6 \pm 1.39^{\mathrm{b}}$ \\
\hline
\end{tabular}

${ }^{1} \mathrm{n}=79$

$a, b, c$ Letras diferentes dentro de filas indican diferencia significativa $(p<0.05)$

Cuadro 5. Correlaciones entre medidas zoométricas de crías de llama

\begin{tabular}{|c|c|c|c|}
\hline Variables & $\begin{array}{l}\text { Observaciones } \\
(\mathrm{n})\end{array}$ & $\begin{array}{c}\text { Correlación } \\
(\mathrm{r})\end{array}$ & $\begin{array}{c}\text { Nivel de } \\
\text { significancia }\end{array}$ \\
\hline Peso corporal - Edad & 171 & 0.725 & 0.009 \\
\hline Peso corporal - Perímetro torácico & 170 & 0.890 & 0.000 \\
\hline Peso corporal - Altura del muslo & 171 & 0.073 & 0.343 \\
\hline $\begin{array}{l}\text { Peso corporal - Perímetro superior del } \\
\text { muslo }\end{array}$ & 171 & 0.117 & 0.127 \\
\hline $\begin{array}{l}\text { Peso corporal - Perímetro inferior del } \\
\text { muslo }\end{array}$ & 171 & -0.099 & 0.197 \\
\hline Edad - Perímetro torácico & 170 & 0.691 & 0.000 \\
\hline Edad - Perímetro superior del muslo & 171 & -0.064 & 0.406 \\
\hline Edad - Perímetro inferior del muslo & 171 & -0.018 & 0.813 \\
\hline Edad - Altura del muslo & 171 & -0.276 & 0.000 \\
\hline $\begin{array}{l}\text { Perímetro torácico - Perímetro superior } \\
\text { del muslo }\end{array}$ & 170 & 0.046 & 0.554 \\
\hline $\begin{array}{l}\text { Perímetro torácico - Perímetro inferior } \\
\text { del muslo }\end{array}$ & 170 & 0.091 & 0.236 \\
\hline Perímetro torácico - Altura del muslo & 170 & -0.078 & 0.312 \\
\hline $\begin{array}{l}\text { Altura del muslo - Perímetro superior del } \\
\text { muslo }\end{array}$ & 171 & 0.484 & 0.000 \\
\hline $\begin{array}{l}\text { Altura del muslo - Perímetro inferior del } \\
\text { muslo }\end{array}$ & 171 & 0.490 & 0.000 \\
\hline $\begin{array}{c}\text { Perímetro superior del muslo- Perímetro } \\
\text { inferior del muslo }\end{array}$ & 171 & 0.855 & 0.000 \\
\hline
\end{tabular}


Cuadro 6. Correlaciones significativas de medidas zoométricas entre madres y crías de llama $^{1}$

\begin{tabular}{|c|c|c|c|c|}
\hline Variable de la madre & $\begin{array}{l}\text { Variable de } \\
\text { la cría }\end{array}$ & $\begin{array}{l}\text { Observaciones } \\
\text { (n) }\end{array}$ & $\begin{array}{l}\text { Correlación } \\
\text { (r) }\end{array}$ & $\begin{array}{c}\text { Nivel de } \\
\text { significancia }\end{array}$ \\
\hline Parto & \multirow{6}{*}{ Peso } & 21 & 0.442 & 0.045 \\
\hline Edad & & 22 & 0.416 & 0.052 \\
\hline Peso & & 22 & 0.375 & 0.086 \\
\hline Ancho de la ubre & & 21 & 0.652 & 0.001 \\
\hline Producción media de leche & & 14 & 0.646 & 0.013 \\
\hline Perímetro área pezones & & 21 & 0.362 & 0.107 \\
\hline Peso & \multirow{6}{*}{$\begin{array}{l}\text { Perímetro } \\
\text { torácico }\end{array}$} & 22 & 0.553 & 0.008 \\
\hline Perímetro torácico & & 21 & 0.388 & 0.082 \\
\hline Ancho de la ubre & & 21 & 0.491 & 0.045 \\
\hline $\begin{array}{l}\text { Perímetro inferior del } \\
\text { muslo }\end{array}$ & & 21 & 0.490 & 0.029 \\
\hline $\begin{array}{l}\text { Perímetro superior del } \\
\text { muslo }\end{array}$ & & 21 & 0.405 & 0.068 \\
\hline Producción media de leche & & 14 & 0.516 & 0.059 \\
\hline Largo de la ubre & \multirow{3}{*}{$\begin{array}{l}\text { Perímetro } \\
\text { superior del } \\
\text { muslo }\end{array}$} & 21 & -0.436 & 0.048 \\
\hline Altura del muslo & & 17 & 0.464 & 0.061 \\
\hline $\begin{array}{l}\text { Perímetro del área de } \\
\text { pezones }\end{array}$ & & 21 & -0.521 & 0.015 \\
\hline Ancho de la ubre & \multirow[b]{2}{*}{$\begin{array}{l}\text { Altura del } \\
\text { muslo }\end{array}$} & 21 & -0.421 & 0.057 \\
\hline $\begin{array}{l}\text { Perímetro del área de } \\
\text { pezones }\end{array}$ & & 21 & 0.451 & 0.040 \\
\hline
\end{tabular}

Las correlaciones entre medidas zoométricas de las madres con mayor valor estadístico se muestran en el Cuadro 3. Destacan las correlaciones de peso corporal con edad, perímetro torácico, perímetro superior e inferior del muslo.

El volumen de producción de leche fue similar en las primeras 7 semanas de lactación
$(252 \pm 85 \mathrm{ml}$ por el periodo de 4 horas de producción). Las correlaciones entre la variable producción de leche y las medidas zoométricas de las madres no tuvieron significancia estadística con excepción de las correlaciones con ancho de la ubre $(\mathrm{p}<0.001)$ y con área del perímetro de los pezones de la ubre y número de partos $(\mathrm{p}<0.1)$. 
En el Cuadro 4 se presenta la estadística descriptiva de las medidas zoométricas de las crías. Las variables peso corporal y perímetro torácico se incrementan con la edad ( $<<0.05)$; sin embargo, los perímetros superior e inferior del muslo no se afectaron por la edad, y la altura del muslo se incrementó significativamente entre los animales de uno a dos meses de edad ( $\mathrm{p}<0.05)$, pero no entre dos a tres meses de edad.

Las correlaciones entre las medidas zoométricas de las crías de las llamas se muestran en el Cuadro 5. Se encontró correlaciones significativas entre la edad y el peso corporal con el perímetro torácico, y entre la altura del muslo con los perímetros superior e inferior del muslo ( $\mathrm{p}<0.0001)$. La correlación entre la edad con la altura del muslo fue inversamente proporcional.

Las correlaciones significativas de medidas zoométricas entre madres y crías se presentan en el Cuadro 6. Las variables de parto, edad, peso, ancho de ubre y producción media de leche se correlacionan en forma positiva con las variable peso y perímetro torácico de las crías.

El perímetro superior e inferior y la altura del muslo de la cría no mostraron correlaciones importantes con las variables de las madres, a excepción de las algunas medidas de la ubre.

\section{Discusión}

El peso de las madres se encuentra, indudablemente, afectado por el número de partos y la edad en la llama (Leyva et al., 1983b; Zea, 2006, Zea et al., 2007) y en la alpaca (Leyva et al., 1983b; Jiménez, 1984), pero en el presente estudio, el peso solamente se afectó en animales viejos (de 5 partos o mayores de 7 años). Esto, evidentemente, representa un factor negativo para determinar criterios de selección, ya que el hecho de no encontrar diferencias entre los estratos de edad y partos (ambas variables asociadas entre sí), disminuye la probabilidad de encontrar variables que se correlacionen entre las variables de las madres. Un comportamiento similar se observó con las variables asociadas al perímetro torácico y a las medidas de la ubre.

Las medidas del muslo se vieron influenciadas por efecto del número de partos, aunque los perímetros superior e inferior del muslo se incrementaron en animales de edad avanzada. Esto indicaría que las diferencias encontradas en el peso de las madres, cuando éstas son de mayor edad, estarían asociadas a un aumento del volumen muscular de sus miembros, sin cambios significativos en la altura del muslo asociado a la madurez alcanzada por el animal adulto en el logro de su máximo tamaño (Condorena, 1980); resultados que dan soporte a los trabajos de Llacsa (2006), Llacsa et al. (2007), Zea (2006) y Zea et al.(2007), donde el volumen del muslo y el peso corporal, aumenta con la edad de las crías.

En base a los resultados de las correlaciones se puede inferir que las variables de las madres que se correlacionan con el peso y que pueden ser utilizadas como criterios de selección son el perímetro torácico, ancho de ubre, altura y perímetros superior e inferior del muslo, y las medidas CB y CD de la grupa. Se dispone de estudios que demuestran correlaciones altas entre peso corporal y perímetro torácico en llamas adultas (Paca, 1977), y entre estas variables y la edad en animales en crecimiento (Llacsa et al., 2007; Zea et al., 2007), lo que indica que el uso del perímetro torácico, una medición de fácil aplicación en el campo, refleja con precisión el peso corporal de la llama. Así mismo, estudios recientes evidencian correlaciones positivas y significativas de peso corporal con el volumen del muslo y el área de la grupa en crías lactantes hasta el destete (Llacsa, 2006; Llacsa et al., 2007) y al año de edad (Zea, 2006; Zea et al., 2007). Estudios afines con correlaciones altas y positivas ( 0.77 y 0.81 ) entre largo de grupa y peso corporal han sido 
encontradas en llamas machos tuis y adultos, respectivamente (Estrada, 1983). Estos resultados permiten recomendar la selección de animales en el hato élite que tengan valores para estas variables por encima de la media del hato, independiente de la edad y del número de partos de la madre.

La producción de leche es una variable importante porque de ella depende la supervivencia de la cría, de allí el interés de seleccionar animales con alto potencial lechero. El ancho de la ubre y el perímetro del área de los pezones son expresiones del tamaño de la ubre y ambos tuvieron una correlación importante con la producción de leche, en concordancia con otros reportes (Llacsa, 2006; Zea, 2006; Llacsa et al., 2007; Zea et al., 2007), aunque también se encuentran reportes que indican la falta de asociación entre producción de leche y tamaño de ubre (Bustinza et al., 1999). Estos resultados concuerdan, además, con trabajos recientes sobre correlaciones altas de producción de leche con peso de la cría (Gonzáles 2006; Gonzáles et al., 2007) y con el ancho de la ubre (Leyva y Gonzáles, en prensa) durante las primeras cuatro primeras semanas de lactación, lo cual indica que el ancho de ubre puede ser un criterio adicional para la selección de animales élite.

Por otro lado, se observa que la producción de leche de las madres no disminuye significativamente conforme transcurren las semanas de lactación. Aún así, se observa una tendencia a una mayor producción de leche durante las 4 primeras semanas de lactación. La leche, en este periodo, es la base de la alimentación de las crías alpacas y llamas (Leyva et al., 1983b; Jiménez, 1984), toda vez que el abomaso es el órgano más desarrollado y activo del aparato digestivo de las crías durante la primera fase de la lactación (Esquerre, 1980) y, por lo tanto, las crías no pueden aprovechar debidamente el forraje (Leyva y Markas, 1991). La producción de leche tiende a disminuir durante la lactación aunque sin cambios de importancia (400 a $250 \mathrm{ml}$ ) por un largo periodo (Leyva, 1990;
Novoa y Leyva, 1996). Estos resultados refuerzan la posibilidad de utilizar el tamaño de ubre como una variable de selección de llamas.

Las variables peso y perímetro torácico de las crías tuvieron un incremento lógico con la edad; sin embargo, las variables perímetro superior e inferior y altura del muslo de las crías no mostraron diferencias significativas con el aumento de la edad, contrastando con los resultados de incrementos significativos del volumen del muslo (que integra los tres medidas en discusión) reportadas en crías de 1 a 8 meses de edad (Llacsa et al., 2007) y de 2 a 15 meses (Zea, 2006; Zea et al., 2007). Probablemente, el rango de edades (de 1 a 3 meses) considerado en el desarrollo de las crías en el presente estudio, no fueron suficientes para mostrar la variabilidad necesaria para encontrar diferencias significativas.

La correlación de las medidas zoométricas entre madre y cría es un indicador de interés para determinar variables para la selección temprana de animales. La primera medición desde el parto hasta 4 semanas después, ofrece información importante, debido a que en ese momento las condiciones medioambientales, sobre todo en la disponibilidad alimenticia y temperatura en el medio andino, son favorables (Proyecto Camélidos Sudamericanos, 1990) y no afectarían las características de las madres y crías por lo que la expresión de los mismos pueden ser atribuidos a los progenitores. Esta selección permite obtener los «mejores animales», los cuales, más adelante, han de expresar sus capacidades dependiendo de las condiciones del medio a los que son expuestos.

Las variables edad y número de parto de la madre no tienen mayor valor para definir criterios de selección temprana. Por otro lado, el peso, ancho de ubre, producción media de leche, e inclusive perímetro del área de los pezones, serían variables de interés para la selección de madres y crías, utilizando en estas últimas el peso como variable de selección, ya que todas ellas están asociadas 
a la producción de leche, sobre todo durante el primer mes de lactación. Asociaciones similares fueron encontradas por Leyva y Gonzales (en prensa). Esto indicaría que una mayor producción de leche aseguraría un mayor probabilidad de obtener crías de mayor peso y con mayor tasa de crecimiento y, como consecuencia, un animal más capacitado para responder a las condiciones del medio ambiente a los que se enfrenta, así como una mayor probabilidad de expresar su carga genética.

Otras correlaciones de utilidad entre madres y crías son el perímetro torácico de la cría con las variables peso, perímetro torácico, ancho de la ubre, perímetro inferior y superior del muslo, producción media de leche y área del perímetro de los pezones de la ubre de la madre. Se debe tener presente que en este y otros estudios (Llacsa et al., 2007; Zea et al., 2007), el perímetro torácico correlaciona fuertemente con el peso de las crías y por tanto puede ser utilizado como criterio de selección adicional.

\section{Conclusiones}

- Las variables asociadas a la producción de leche (largo, ancho y área del perímetro de los pezones de la ubre) son las más importantes para determinar crías de mejor peso $\mathrm{y}$, por lo tanto, deben ser consideradas como criterio de selección de las madres para obtener crías de alto valor fenotípico.

- Las variables peso de la madre, y perímetro superior e inferior del muslo deben de ser, además, incluidas para la selección de animales élite.

- En una primera selección se debería mantener en el hato élite a todas aquellas madres cuyos valores para las variables indicadas se encuentren por encima del promedio del hato.

\section{Literatura Citada}

1. Bravo, W.; E. Franco; J. Sumar. 1981. Rendimiento de canal en llamas. IV Convención Internacional sobre Camélidos Sudamericanos. Punta Arenas, Chile.

2. Bustinza, V.; J. Garnica; Z. Maquera; G. Medina; E. Apaza; S. Foraquita; O. Carrión. 1993. Carne de alpaca. Escuela de Postgrado, Universidad Nacional del Altiplano. Puno- Perú.

3. Bustinza, V.; V. Ibáñez; J. Jaen; A. Astete. 1999. Tamaño de ubre. II Congreso Mundial sobre Camélidos Sudamericanos. Cuzco, Perú.

4. Calderón, W.; S. Fernández-Baca. 1972. Peso vivo y rendimiento de canal en la alpaca. Rev. Inv. Pec. (IVITA) 1(1): 5-9.

5. Condori, G.; C. Ayala; C. Renieri; T. Rodríguez; Z. Martínez. 2003. Alometría de cortes comerciales en carcasa de llama en dos fases de crecimiento. III Congreso Mundial sobre Camélidos Sudamericanos. Potosí, Bolivia. p 619-626.

6. Condorena, N. 1980. Algunos índices de producción de alpacas bajo sistema de la esquila anual establecido en la Raya. Rev. Inv. Pec. (IVITA) 5(1): 50-54.

7. Esquerre, C. 1980. Contenido relativo de digesta gastrointestinal de la alpaca adulta. VI Congreso Nacional de Ciencias Veterinarias. Piura, Perú. p 25-26.

8. Estrada, A. 1983. Zoometría y algunas correlaciones en llamas. Tesis de Ingeniero Zootecnista, Universidad San Antonio Abad del Cusco. Cusco, Perú. 81 p.

9. Fernández-Baca, S. 1961. Ensayo de engorde de ovinos en la altura. Rev. Fac. Med. Vet. UNMSM 16-17: 109-127.

10. Gonzáles, R. 2006. Efecto de dos niveles nutricionales sobre la producción láctea en llamas seleccionadas para producción de carne. Tesis de Médico Ve- 
terinario. Facultad de Medicina Veterinaria, Univ. Nacional Mayor de San Marcos. Lima. 52 p.

11. Gonzáles, R., V. Leyva; W. García; $C$. Gaviria; D. Ticona. 2007. Efecto de la alimentación sobre la producción láctea en llamas seleccionadas para producción de carne. Rev. Inv. Vet., Perú 18: 30-39.

12. Jiménez, J. 1984. Efecto de la edad sobre la producción de leche de alpacas bajo condiciones de pastura natural. Tesis de Bachiller en Medicina Veterinaria. Facultad de Medicina Veterinaria, Univ. Nacional Mayor de San Marcos. Lima. 32 p.

13. Johnson, L.W. 1994. Llama nutrition. Review: Update on llama medicine. Vet. Clin. N. Am. Food Anim. Pract. 10: 187201.

14. Leyva, V. 1989. Sistemas de producción de alpacas. En: Simposio Producción de Alpacas y Llamas. p 157-168. XII Reunión Asoc. Per. Prod. Anim. Lima.

15. Leyva, V. 1990. Informe de la VIII Reunión de Trabajo sobre Sistemas de Producción Animal. Centro de Investigación Internacional para el Desarrollo (CIID). MR139s: 143-169.

16. Leyva, V.; E. Franco; N. Condorena. 1983a. Evaluación de dos técnicas para estudios sobre la lactación en Camélidos Sudamericanos. VI Reunión Asoc. Per. Prod. Anim. Lambayeque, Perú.

17. Leyva, V.; E. Franco; N. Condorena. 1983b. Patrón lactacional de alpacas y llamas bajo condiciones de pastura natural. VI Reunión Asoc. Per. Prod. Anim. Lambayeque, Perú.

18. Leyva, V.; J. Markas. 1991. Involución de la glándula mamaria en alpacas y efecto sobre el peso corporal y producción de fibra. Turrialba 41(1): 59-63.
19. Llacsa, J. 2006. Determinación de indicadores biométricos para la selección de llamas (Lama glama) productoras de carne. Tesis de Médico Veterinario y Zootecnista. Facultad de Medicina Veterinaria y Zootecnia, Univ. Nacional del Altiplano. Puno, Perú. 49 p.

20. Llacsa, J.; M. Urviola; V. Leyva. 2007. Evaluación de indicadores biométricos en llamas (Lama glama) de las variedades Ch'acu y K'ara. Rev. Inv. Vet., Perú 18: 1-10.

21. Novoa, C.; V. Leyva. 1996. Reproducción en alpacas y llamas. Publ. Cient. IVITA N ${ }^{\circ}$. 26. UNMSM. Lima. 30 p.

22. Proyecto Camélidos Sudamericanos. 1990. Informe Técnico Fase III del Convenio IVITA- UNMSM y Centro Internacional de Investigación para el Desarrollo. UNMSM. Lima. 57 p.

23. Paca, M. 1977. Biometría de la llama (Lama glama). Tesis de Médico Veterinario y Zootecnista. Facultad de Medicina Veterinaria y Zootecnia, Univ. Nacional del Altiplano. Puno, Perú. 39 p.

24. Pugh, D.G. 1997. Nutrition and feeding of South American camelids. Proc. Central Veterinary Conference. Kansas City, Mo. p 150-153.

25. San Martín, H. 1996. Nutrición en alpacas y llamas. Pub. Cient. IVITA No. 27. UNMSM. Lima. 29 p.

26. SAS. 1996. The SAS System for windows. v 6.12. User's guide. SAS Institute Inc. $889 \mathrm{p}$.

27. Zea, O. 2006. Evaluación de las medidas del área de la grupa y volumen del muslo y de la ubre para uso como indicadores genéticos en la selección temprana de llamas (Lama glama) para carne. Tesis de Médico Veterinario. Facultad de Medicina Veterinaria, Univ. Nacional Mayor de San Marcos. Lima. 86 p. 